\title{
Attention to Context during Evaluative Learning and Context-Dependent Automatic Evaluation: A Cross-Cultural Analysis
}

\author{
Yang Ye ${ }^{1,2}$, Yuk-Yue Tong ${ }^{3}$, Chi-Yue Chiu ${ }^{4}$, Bertram Gawronski ${ }^{2,5}$ \\ ${ }^{1}$ Ghent University, Belgium \\ ${ }^{2}$ University of Western Ontario, Canada \\ ${ }^{3}$ Singapore Management University, Singapore \\ ${ }^{4}$ The Chinese University of Hong Kong, Hong Kong, China \\ ${ }^{5}$ University of Texas at Austin, USA
}

\begin{abstract}
Previous research has shown that changes in automatic evaluations can be limited to the context in which counterattitudinal information was acquired. This effect has been attributed to enhanced attention to context cues during the encoding of expectancy-violating counterattitudinal information. Drawing on previous evidence for cultural differences in attention to context and tolerance for inconsistency, the present research examined cultural differences in responses to conflicting evaluative information and the resulting contexteffects on automatic evaluation. Study 1 revealed that both Canadian and Singaporean participants showed enhanced attention to context during exposure to counterattitudinal information. In a reanalysis of studies with Singaporean participants, Study 2 replicated the pattern of contextualized changes of automatic evaluations previously obtained in Western participants. The results suggest that contextualized change of automatic evaluations might be a general phenomenon that replicates across cultures. Implications for East-West similarities in basic attentional processes and automatic evaluation are discussed.
\end{abstract}

Keywords: attitude change; automatic evaluation; attention; context effects; culture

Counter to earlier research suggesting that automatic evaluations are relatively difficult to change, an accumulating body of evidence suggests that automatic evaluations can change rapidly in response to counterattitudinal information (for a review, see Gawronski \& Bodenhausen, 2006). To reconcile the conflicting findings, it has been argued that changes in automatic evaluations can be limited to the context in which the counterattitudinal information was learned (Brannon \& Gawronski, in press; Gawronski, Rydell, Vervliet, \& De Houwer, 2010; Gawronski, Ye, Rydell, \& De Houwer, 2014; Rydell \& Gawronski, 2009). In their representational theory, Gawronski et al. (2010) specified the processes by which context cues become integrated into the representation of conflicting evaluative information about an object, which allows these cues to moderate automatic evaluations upon future encounters with that object (for a review, see Gawronski \& Cesario, 2013). Despite the large body of evidence supporting the theory (for a meta-analysis, see Gawronski, Hu, Rydell, Vervliet, \& De Houwer, 2015), most studies were conducted with samples from Western cultures. Because research in cross-cultural psychology suggests possible East-West differences in the context-effects predicted by the representational theory (Ye \& Gawronski, in press), it seems imperative to investigate the generality of these effects across cultures. By examining potential cultural differences in the hypothesized context-effects, the present research aims to deepen our understanding of cultural influences on (1) fundamental psychological processes involved in evaluative learning and (2) social impression formation in situations that involve conflicting information.

\section{The Representational Theory}

According to Gawronski et al.'s (2010) representational theory, attention to context during the learning of evaluative information determines whether context cues are integrated into the representation of the newly acquired information. If attention to context during the learning of evaluative information is high, the newly acquired information is assumed to be stored in a contextualized representation. Yet, if attention to context during the learning of evaluative information is low, the newly acquired information should be stored in a context-free representation. The theory further assumes that attention to context is typically low during the learning of initial attitudinal information (Gilbert \& Malone, 1995) and enhanced by exposure to expectancy-violating counterattitudinal information (Roese \& Sherman, 2007). As a result, initial attitudinal information tends to be stored in context-free representations, whereas expectancy-violating counterattitudinal information is stored in contextualized representations. Together with the principle of feature-matching in memory activation (Smith, 1996), these assumptions imply that counterattitudinal information should influence automatic evaluations only in the context in which this information was learned. In contrast, initial attitudinal information should determine automatic evaluations in any other context, including the context in which the initial attitudinal information was learned and any other 
context in which the target objects had not been encountered before.

To describe the context-effects resulting from these processes, Gawronski et al. (2010) adapted the term renewal effect from animal learning research (see Bouton, 2004). ABA renewal refers to cases in which initial attitudinal information is learned in Context $A$, counterattitudinal information is subsequently learned in a different Context B, and the initial attitudinal information determines automatic evaluations in the initial Context A. Correspondingly, $A B C$ renewal refers to cases in which initial attitudinal information is learned in Context A, counterattitudinal information is subsequently learned in a different Context B, and the initial attitudinal information determines automatic evaluations in a novel Context $C$. These patterns differ from automatic evaluations in $\mathrm{ABB}$ scenarios in which initial attitudinal information is learned in Context A, counterattitudinal information is subsequently learned in a different Context B, and the counterattitudinal information determines automatic evaluations in Context B. Consistent with the predictions of their representational theory, several studies by Gawronski and colleagues (Gawronski et al., 2010, 2014; Rydell \& Gawronski, 2009) found reliable evidence for ABA and $A B C$ renewal effects on automatic evaluations of a target person when participants had learned conflicting evaluative information about this person (i.e., statements about positive and negative behaviors) in different contexts (i.e., the statements being presented against different colored backgrounds).

\section{A Cross-Cultural Analysis}

Although Gawronski et al. (2010) treated the reviewed pattern of context-effects as the default outcome, their theory also implies specific predictions for two alternative scenarios involving different levels of attention to context during learning. First, if attention to context is high during the learning of initial attitudinal information, attitudinal and counterattitudinal information should be stored in two contextualized representations. In this case, $A B C$ renewal should be reduced, because encountering the target in a novel context should activate the two contextualized representations to the same extent, producing automatic evaluations that reflect the average of the two types of information. Yet, ABA renewal should be unaffected, because encountering the target in the context of the initial attitudinal information should activate the contextualized representation of that information. This prediction has been confirmed in a study by Gawronski et al. (2010) in which attention to context during the learning of initial attitudinal information was experimentally enhanced.

Second, if attention to context is low during the learning of counterattitudinal information, attitudinal and counterattitudinal information should be integrated in a single context-free representation. In this case, both $\mathrm{ABA}$ and $\mathrm{ABC}$ renewal should be reduced, because encountering the target should activate this integrated, context-free representation regardless of the context. This prediction has been confirmed in a study by Gawronski et al. (2010) in which attention to context during the learning of counterattitudinal information was experimentally reduced.

Expanding on the two scenarios, Ye and Gawronski (in press) proposed that they represent two possible ways in which individuals from Eastern and Western cultures may differ in the learning of conflicting evaluative information, and thus in the tendency to show $\mathrm{ABA}$ and $\mathrm{ABC}$ renewal. ${ }^{1}$ First, drawing on research showing that Easterners generally pay more attention to context than Westerners (e.g., Chua, Boland, \& Nisbett, 2005; Masuda \& Nisbett, 2001; Masuda, Russell, Chen, Hioki, \& Caplan, 2014; Zhou, He, Yang, Lao, \& Baumeister, 2012), it is possible that Easterners pay more attention to context during the learning of initial attitudinal information (Figure 1, Hypothesis 1). Consequently, Easterners should show weaker effects of $\mathrm{ABC}$ renewal and similar effects of ABA renewal compared to Westerners. Second, drawing on research showing that dialectical thinking is more prevalent among Easterners whereas analytical thinking is more prevalent among Westerners (Jenkins, Yang, Goh, Hong, \& Park, 2010; Peng \& Nisbett, 1999; Spencer-Rodgers, Williams, \& Peng, 2010), it is possible that Easterners pay less attention to context during the learning of counterattitudinal information than Westerners (Figure 1, Hypothesis 2). This hypothesis is based on the notion that a given object can have opposite attributes from dialectical view, which would represent a logical contradiction from an analytical view. Therefore, dialectical thinking can weaken Easterners' perception of inconsistency (see Choi \& Nisbett, 2000), the driving force behind enhanced attention to context during the learning of counterattitudinal information (Gawronski, 2012). Consequently, Easterners should show weaker effects of ABA and ABC renewal compared to Westerners.

Gawronski et al. (2015) recently conducted a metaanalysis of all studies from their research groups that

\footnotetext{
${ }^{1}$ For the sake of brevity, we refer to individuals from the two cultures as Easterners and Westerners. This demarcation is not intended to imply perfect homogeneity within cultures. For example, although explanations of social events in terms of contextual factors are more prevalent in Eastern than Western cultures, there is considerable variability in causal explanations within cultures (Chiu \& Hong, 2006).
} 
tested predictions of Gawronski et al.'s (2010) representational theory. Because the meta-analysis included a small number of unpublished studies conducted in Singapore, the findings provide preliminary evidence for the current question of whether Easterners and Westerners differ with regard to their susceptibility to $\mathrm{ABA}$ and $\mathrm{ABC}$ renewal. The most relevant finding is that effect sizes of $\mathrm{ABA}$ and $\mathrm{ABC}$ renewal significantly differed from zero in samples from the United States and Canada, but not in samples from Singapore.

Although this result seems to support Hypothesis 2, the possibility of strong conclusions is undermined by the lack of a significant difference between Singaporean and Canadian participants, the latter of which showed significant effects for both $\mathrm{ABA}$ and $\mathrm{ABC}$ renewal. Another limitation is that the absence of $\mathrm{ABA}$ and $\mathrm{ABC}$ renewal in Singaporean participants might reflect a simple replication failure due to sampling or measurement error (Maxwell, Lau, \& Howard, 2015; Stanley \& Spence, 2014). After all, the meta-analysis also included several studies that failed to replicate the predicted patterns of ABA and ABC renewal in Western samples. Thus, to allow for stronger conclusions, it is critical to provide independent evidence for cultural differences in the hypothesized processes and the resulting context-effects on automatic evaluations.

\section{Study 1}

Study 1 tested the proposed East-West difference in attention to context during the learning of counterattitudinal information. Adapting a paradigm from Gawronski et al. (2014, Experiments 1a \& 1b), participants from Canada and Singapore received either positive or negative information about an unknown target individual and were then exposed to information that was either congruent or incongruent with the valence of the initial information. To measure attention to incidental context cues, the statements about the target individual were presented against randomly varying background colors. After the impression formation task, participants completed a surprise recognition test in which they were asked to indicate the background color against which the critical target statement had been presented. The idea underlying this paradigm is that expectancy-violations resulting from inconsistent information enhance attention to the context, which should improve participants' memory for the incidental background color (cf. Cacioppo, Crites, Berntson, \& Coles, 1993). Thus, to the extent that Singaporean participants pay less attention to the context of counterattitudinal information, they should show a reduced memory advantage for the context of expectancy-incongruent over expectancy-congruent information compared to Canadian participants. ${ }^{2}$

\section{Method}

Participants and design. One-hundred Canadian undergraduate students from the University of Western Ontario (78 women and 22 men, mean age 18.7 years) participated for a course requirement and 80 Singaporean undergraduate students from the Singapore Management University (50 women and 30 men, mean age 21.4 years) participated for SG\$5 (about US\$3.6). ${ }^{3}$ The study included a 2 (Country: Canada vs. Singapore) $\times 2$ (Valence of Initial Information: positive vs. negative) $\times 2$ (Target Statement: congruent with initial information vs. incongruent with initial information) between-subjects design.

Impression formation task. Participants were asked to form an impression of a target person based on behavioral information (Gawronski et al., 2014, Experiments 1a \& 1b). Toward this end, participants were presented with 30 behavioral statements along with a picture of the person's face. To obtain an ethnically ambiguous face, we created a 50\% morph of a face-pair that included an Asian man and a Caucasian man. The statements were adapted from Rydell and Gawronski (2009) with minor adjustments to ensure that they are perceived as equally positive and negative in Eastern and Western cultures. ${ }^{4}$ The valence and cultural independence of the statements was confirmed in a pretest. The first 20 and the last 9 statements were of the same valence and randomly selected from two lists of 29 positive or 29 negative statements; the 21st statement served as the critical target statement that was either congruent or incongruent with the valence of the other statements. Each statement was presented for 5000ms against one of ten colored backgrounds. The screen

\footnotetext{
${ }^{2}$ We aimed to recruit at least 80 participants from each country. Based on the average effect size for mean level differences in earlier research using the same paradigm (Gawronski et al., 2014), a sample of 80 participants provides a power of .859 to detect an expectancyviolation effect within each group. The data were collected in one shot without prior statistical analyses. We report all data exclusions, all experimental manipulations, and all dependent measures. All materials and data are available from the authors upon request.

${ }^{3}$ Of the 100 participants in the Canadian sample, 27 were reported being born in Asia. Excluding these participants did not change the pattern and statistical significance of the reported results. Of the 80 participants in the Singaporean sample, none was born in North America.

${ }^{4}$ Because English is one of the main languages in Singapore and the primary language at the university from which we recruited our participants, all materials were presented in English.
} 
turned black during the 1000ms inter-trial interval. Each colored background appeared once during one of three 10-trial blocks. The critical target statement was always presented against a blue background. There was no reference to the background in the task instructions.

Recognition test. After the impression formation task, participants completed a 7-item surprise recognition test. Each trial showed 10 squares, displaying the 10 background colors (numbered from 0 to 9), and one of the statements from the impression formation task. Participants were asked to identify the background color against which each statement had been presented in the impression formation task. The first item included the critical target statement; the following six items included statements that were randomly selected from the other 29 statements. Participants' memory performance on the critical target item served as the primary dependent measure.

Results

Data were analyzed with binary logistic regression. The three independent variables Country (Canada vs. Singapore), Valence of Initial Information (positive vs. negative), and Target Statement (incongruent vs. congruent) were dummy-coded and entered simultaneously into the regression model together with all possible interactions. The analysis revealed a significant main effect of Target Statement, $B=1.94$, $S E=0.87$, Wald $=4.94, p=.026, O R=6.96$, indicating that memory for the background color was better when the target statement was incongruent $(M=.39$, CI $[.30, .47])$ than when it was congruent $(M=.08$, CI [$.009, .16]$ ) with the initial attitudinal information (see Figure 2). No other main or interaction effect reached statistical significance (all ps $>.61$ ), including the twoway interaction between Country and Target Statement $(p=.62)$. Analyses within each group of Country revealed that the effect of Target Statement was significant for both Canadian participants, $B=1.46$, $S E$ $=0.57$, Wald $=6.57, p=.010, O R=4.31$, and Singaporean participants, $B=2.72, S E=0.80$, Wald $=$ 11.63, $p=.001, O R=15.10$. If anything, Singaporean participants showed a stronger memory advantage for the background of incongruent information than Canadian participants.

\section{Discussion}

The findings of Study 1 contradict the hypothesis that individuals from Eastern countries pay less attention to context during the learning of counterattitudinal information (Ye \& Gawronski, in press). Thus, they are at odds with the proposed interpretation of Gawronski et al.'s (2015) meta-analytic findings, which showed $\mathrm{ABA}$ and $\mathrm{ABC}$ renewal in samples from the United States and Canada, but not in samples from Singapore. This conclusion raises the question of what accounts for the lack of $\mathrm{ABA}$ and $\mathrm{ABC}$ renewal in Singaporean samples in Gawronski et al.'s (2015) meta-analysis.

One possibility is that the absence of $\mathrm{ABA}$ and $\mathrm{ABC}$ renewal in Singaporean samples reflects a simple replication failure as a result of measurement error (Maxwell et al., 2015; Stanley \& Spence, 2014). Consistent with the latter interpretation, a closer inspection of Gawronski et al.'s (2015) meta-analysis suggests that effect sizes for the Singaporean samples might have been attenuated by the low reliability of one of the employed measures. Although the meta-analysis revealed significant renewal effects for various measures of automatic evaluation, there were no significant effects for studies that used the evaluative priming task as a measure of automatic evaluation (EPT; Fazio, Jackson, Dunton, \& Williams, 1995). Because the EPT tends to show rather low estimates of reliability (Cronbach's Alpha values between .00 and .55; see Gawronski \& De Houwer, 2014), it is possible that the failure to obtain significant renewal effects with the EPT is due to substantial proportions of measurement error (LeBel \& Paunonen, 2011). Consistent with this possibility, studies that utilized the affective misattribution procedure (AMP; Payne, Cheng, Govorun, \& Stewart, 2005), a measure of automatic evaluation known for its high reliability (Cronbach's Alpha values between .70 and .90; see Gawronski \& De Houwer, 2014), showed meta-analytic effect sizes that were (1) significantly larger and (2) significantly different from zero.

Of the three studies with Singaporean samples in Gawronski et al.'s (2015) meta-analysis, one used the EPT and two used the AMP as a measure of automatic evaluation. This ratio is higher than compared to the ratio in the entire sample, where only 4 out of 30 studies used the EPT as a dependent measure. Hence, it is possible that the absence of $\mathrm{ABA}$ and $\mathrm{ABC}$ renewal in Singaporean samples was due to the relatively large impact of a single study with an unreliable measure rather than genuine cultural differences in attention to context during the learning of counterattitudinal information.

\section{Study 2}

The main goal of Study 2 was to re-examine the emergence of ABA and ABC renewal in Singaporean samples while controlling for potential confounds with measurement error. Toward this end, we obtained an updated Singaporean sample that included all studies that we have conducted in Singapore except for one study that used the EPT. Our data base for this approach included three identical replications that used the AMP as a dependent measure. Two of these studies had already been included in the meta-analysis; one additional study was conducted shortly after Gawronski et al.'s (2015) meta-analysis was accepted for publication. This updated sample allowed us to draw 
stronger conclusions about the emergence of $\mathrm{ABA}$ and $\mathrm{ABC}$ renewal in Eastern cultures.

\section{Method}

Participants and design. Study 2 combined the samples from three independent studies: 103 students from the Singapore Management University (67 women, 36 men, mean age $=21.1$ ), 102 students from the Nanyang Technological University (51 women, 51 men, mean age $=20.9$ ), and 101 students from the Singapore Management University (57 women, 42 men, 2 unspecified, mean age $=21.6)$. All three studies used the same 2 (Background Order: yellow-blue vs. blue-yellow, between-subjects) $\times 2$ (Valence Order: positive-negative vs. negative-positive, within-subjects) $\times 3$ (Evaluation Background: yellow vs. blue vs. green, within-subjects) mixed-model design (see Gawronski et al., 2014, Experiment 2). Participants received research credit for an introductory psychology course or SG\$5 (US\$3.6). With the average effect sizes obtained in Gawronski et al.'s (2015) meta-analysis, the updated sample $(N=306)$ provided a power of .991 to detect an ABA renewal effect and a power of .859 to detect an ABC renewal effect.

Impression formation task. Participants were asked to form first impressions of two individuals on the basis on behavioral information (see Gawronski et al., 2014, Experiment 2). Each target was depicted with a picture of an ethnically ambiguous man (see Study 1 ). In the first block, participants were presented with 20 positive behaviors performed by one of the two individuals and 20 negative behaviors performed by the other individual. In the second block, participants were presented with 40 new behavioral statements, such that the target paired with positive statements in the first block was now paired with negative statements, and vice versa. The statements were adapted from the same set of pretested statements used in Study 1. Each picture-statement pair was presented for $5000 \mathrm{~ms}$ against a colored background, which remained on the screen throughout each block, with an inter-trial interval of $1000 \mathrm{~ms}$. A different background color (blue vs. yellow) was used for each block. The background color for each block and the valence of information about each target individual were counterbalanced. The order of trials was randomized.

Automatic evaluation. Following the impression formation task, automatic evaluations were assessed with the AMP. On each trial, participants were presented with a prime stimulus (i.e., face of one of the two individuals) for $100 \mathrm{~ms}$, a blank screen for $100 \mathrm{~ms}$, and an ambiguous polygon for $100 \mathrm{~ms}$, which was followed by a black-and-white pattern mask. Participants were asked to indicate if they considered the polygon visually more pleasant or visually less pleasant than average by pressing one of two response keys. The AMP consisted of 120 trials, including 60 trials for each of the two faces. To investigate effects of the background colors on automatic evaluations, we manipulated the background color during the $100 \mathrm{~ms}$ presentation of the face primes, with one-third of the trials showing the background of the first learning block, one-third showing the background of the second learning block, and the remaining third showing a novel background that had not been presented in the impression formation task (i.e., green). Following Payne et al. (2005), participants were told that the pictures can influence their evaluations of the polygons and that they should try their absolute best to avoid any biasing influence.

\section{Results}

AMP responses were aggregated by calculating the proportion of more pleasant responses for each target individual against each of the three backgrounds. Scores were collapsed across conditions to reflect (1) the order of evaluative statements about each target (positivenegative vs. negative-positive), and (2) the nature of the backgrounds with reference to the two blocks of the impression formation task (first context vs. second context vs. novel context). The resultant scores were submitted to a 2 (Valence Order: positive-negative vs. negative-positive) $\times 3$ (Evaluation Context: first vs. second vs. novel) repeated-measures ANOVA, which revealed a significant two-way interaction of Valence Order and Evaluation Context, $F(2,608)=6.25, p$ $=.002, \eta_{\mathrm{p}}{ }^{2}=.020$ (see Figure 3 ). No other effects reached statistical significance (all $F \mathrm{~s}<0.98$, all $p s>$.32). To decompose this interaction, we conducted a priori pairwise comparisons reflecting the emergence of ABA and ABC renewal (see Gawronski et al., 2015).

For ABA renewal, automatic evaluations assessed in the first context (Context A) were compared to those in the second context (Context B) at each level of Valence Order. In the positive-negative condition, we found a significant $\mathrm{ABA}$ renewal effect, in that automatic evaluations were more positive in Context A than Context B, $t(304)=2.72, p=.007, d=0.16$. In the negative-positive condition, automatic evaluations seemed more negative in Context A than in Context B, but the difference was not statistically significant, $t(304)$ $=1.07, p=.29, d=0.061$. To investigate potential order effects, we calculated indices of the size of ABA renewal effects for each Valence Order condition by subtracting AMP scores in Context B from AMP scores in Context $\mathrm{A}$ in the positive-negative condition, and vice versa in the negative-positive condition. The resulting scores were submitted to an ANOVA with Valence Order as a within-subjects factor. The analysis revealed no significant effect of Valence Order, $F(1$, $304)=1.03, p=.31, \eta_{\mathrm{p}}{ }^{2}=.003$. Yet, the analysis did reveal a significant intercept, $F(1,304)=7.24, p=.008$, $\eta_{\mathrm{p}}{ }^{2}=.023$, indicating an overall ABA renewal effect. 
For ABC renewal, automatic evaluations assessed in the novel context (Context $\mathrm{C}$ ) were compared to those in the second context (Context B) in the manner described for ABA renewal. In the positive-negative condition, the results indicated a significant $A B C$ renewal effect, in that automatic evaluations were more positive in Context $\mathrm{C}$ than Context $\mathrm{B}, t(304)=2.22, p$ $=.027, d=0.13$. Conversely, in the negative-positive condition, automatic evaluations were marginally more negative in Context $\mathrm{C}$ than Context $\mathrm{B}, t(304)=1.96, p$ $=.051, d=0.11$. To investigate potential order effects, we calculated indices of the size of ABC renewal effects for each condition of Valence Order by subtracting AMP scores in Context B from AMP scores in Context $\mathrm{C}$ in the positive-negative condition, and vice versa in the negative-positive condition. An ANOVA with Valence Order as a within-subjects factor revealed no significant effect of Valence Order, $F(1,304)=0.00, p$ $=1.00, \eta_{\mathrm{p}}{ }^{2}=.00$. Yet, the ANOVA did reveal a significant intercept, $F(1,304)=10.40, p=.001, \eta_{\mathrm{p}}{ }^{2}$ $=.033$, indicating an overall ABC renewal effect.

To investigate potential differences in the size of $\mathrm{ABA}$ and $\mathrm{ABC}$ renewal effects, aggregate scores of $A B A$ and $A B C$ renewal were submitted to a 2 (Effect Type: $A B A$ renewal vs. ABC renewal) $\times 2$ (Valence Order: positive-negative vs. negative-positive) repeatedmeasures ANOVA. This analysis revealed no significant main or interaction effects (all $F$ s $<1.53$, all ps $>$.21). Post-hoc comparisons indicated no significant difference between the size of ABA renewal and that of $\mathrm{ABC}$ renewal in the positive-negative condition, $t(304)$ $=0.72, p=.47, d=0.041$, and in the negative-positive condition, $t(304)=-1.08, p=.28, d=-0.062$.

\section{Discussion}

Study 2 obtained evidence for both ABA and ABC renewal in Singaporean participants. Replicating earlier findings with North American samples (e.g., Gawronski et al., 2010, 2014; Rydell \& Gawronski, 2009), automatic evaluations reflected the valence of counterattitudinal information only in the context in which this information was learned. In contrast, automatic evaluations reflected the valence of the initial attitudinal information in other contexts, including the context of the attitudinal information and a novel context in which the target had not been encountered before. Together, these results suggest that contextualized change of automatic evaluations is a general phenomenon that replicates across cultures. ${ }^{5}$

\footnotetext{
${ }^{5}$ As an additional test of cross-cultural differences in ABA and $A B C$ renewal, we compared the effect sizes in Study 2 to the meta-analytic effect sizes in studies with Western participants using the same
}

\section{General Discussion}

Drawing on Gawronski et al.'s (2010) representational theory and research in cross-cultural psychology, we tested two hypotheses on East-West differences in the encoding of conflicting evaluative information and their downstream effect on automatic evaluations (see Figure 1). According to the first hypothesis, Easterners pay more attention to context during the learning of initial attitudinal information than Westerners, and therefore show weaker effects of ABC renewal. According to the second hypothesis, Easterners pay less attention to context during the learning of counterattitudinal information than Westerners, and therefore show weaker effects of both $A B A$ and $A B C$ renewal. Although a recent meta-analysis (Gawronski et al., 2015) seemed to support the second hypothesis, the current findings contradict both hypotheses. Both Easterners and Westerners showed (1) enhanced attention to context during the encoding of expectancyviolating counterattitudinal information and (2) contextualized changes in automatic evaluations after learning conflicting evaluative information.

The current findings seem to contradict previous evidence showing that Easterners generally pay more attention to context (e.g., Chua et al., 2005; Zhou et al., 2012) and have a higher for tolerance of inconsistency (e.g., Peng \& Nisbett, 1999; Spencer-Rodgers et al., 2010) than Westerners. To reconcile these discrepancies, it is important to note two fundamental differences between the present and previous research. First, whereas previous research on attention to context focused on contexts that have a clear conceptual relation to the learned information, the present research was concerned with incidental context cues that are irrelevant for the learned information. Second, whereas previous research on tolerance of inconsistency focused mainly on how inconsistency influences thinking and reasoning, the present research was concerned with the effect of conflicting information on automatic evaluations. Together, these differences highlight the

procedural details (i.e., within-subject manipulation of valence-order and use of a measure other than the EPT; see Gawronski et al., 2015). In the current study, the average effect size for ABA renewal $(d=0.11)$ was outside the $95 \%$ confidence interval of the meta-analytic effect size $(d=0.200$, CI $[0.157,0.243])$, and that for ABC renewal $(d=$ 0.12 ) was inside the $95 \%$ confidence interval of the meta-analytic effect size $(d=0.121$, CI $[0.076,0.166])$. Although these findings suggest potential cultural differences in ABA renewal, they are incompatible with both of our hypotheses (also see Ye \& Gawronski, in press). Because these comparisons may involve unrecognized confounds, future research is needed to establish the reliability of the obtained difference in ABA renewal. 
contribution of the present research by showing that, in contrast to the well-documented differences in controlled processing, Easterners and Westerners may be relatively similar in terms of automatic processing.

The present research also makes a valuable contribution to the current debate about the replicability of social psychological findings (Open Science Collaboration, 2015). First, our findings echo LeBel and Paunonen's (2011) concern that unreliable measurement can reduce the likelihood of replicating an actually existing effect. To maximize the informational value of replication studies, it is essential to ensure reliable measurement of the critical outcome. Second, our findings illustrate the significance of culture in understanding successful and failed replications. As Van Bavel, Mende-Siedlecki, Brady, and Reinero (2016) pointed out, the success of direct replications across cultures may depend on the culturally specific meanings of materials, even if the hypothesis about the mechanism underlying the to-be replicated effect is correct (cf. Kashima, 2015). From this perspective, the present research makes a valuable contribution by demonstrating the cross-cultural replicability of two key findings despite the existence of theoretical arguments for cultural differences.

Given the strong influence of Western culture on the Singaporean society, one could argue that Singapore may not be the best candidate for a study on crosscultural differences in attention and thinking styles. Another potential objection is that our findings might have been distorted by the use of English materials, given that language can moderate the use of culturespecific thinking styles in participants with bi-cultural identity (for a review, see Oyserman \& Lee, 2008). Both concerns can be alleviated with a study by Ji, Zhang, and Nisbett (2004), who found that ethnically Chinese participants from Singapore showed the same relational reasoning style as participants from other regions in East Asia (e.g., Taiwan, mainland China, Hong Kong). Importantly, unlike participants from mainland China or Taiwan, the relational thinking style of Singaporean Chinese was unaffected by the use of English or Chinese, making them ideal candidates for testing EastWest differences. Nevertheless, future research should explore whether the current findings generalize to individuals from other Eastern countries.

In sum, the present research suggests individuals from Eastern and Western cultures may be relatively similar in terms of their susceptibility to contextualized changes of automatic evaluations and the attentional processes underlying such changes. Thus, in addition to the well-documented differences in reasoning and thinking, there might be an interesting layer of cultural similarities in automatic processes awaiting further research.

\section{References}

Bouton, M. E. (2004). Context and behavioral processes in extinction. Learning and Memory, 11, 485-494.

Brannon, S. M., \& Gawronski, B. (in press). A second chance for first impressions? Exploring the context (in)dependent updating of implicit evaluations. Social Psychological and Personality Science.

Cacioppo, J. T., Crites, S. L., Jr., Berntson, G. G., \& Coles, M. G. H. (1993). If attitudes affect how stimuli are processed, should they not affect the event-related brain potential? Psychological Science, 4, 108-112.

Chiu, C.-Y., \& Hong, Y. (2006). Social psychology of culture. New York: Psychology Press.

Choi, I., \& Nisbett, R. E. (2000). Cultural psychology of surprise: Holistic theories and recognition of contradiction. Journal of Personality and Social Psychology, 79, 890-905.

Chua, H. F., Boland, J. E., \& Nisbett, R. E. (2005). Cultural variation in eye movements during scene perception. Proceedings of the National Academy of Sciences of the United States of America, 102, 12629-12633.

Fazio, R. H., Jackson, J. R., Dunton, B. C., \& Williams, C. J. (1995). Variability in automatic activation as an unobtrusive measure of racial attitudes: A bona fide pipeline? Journal of Personality and Social Psychology, 69, 1013-1027.

Gawronski, B. (2012). Back to the future of dissonance theory: Cognitive consistency as a core motive. Social Cognition, 30, 652-668.

Gawronski, B., \& Bodenhausen, G. V. (2006). Associative and propositional processes in evaluation: An integrative review of implicit and explicit attitude change. Psychological Bulletin, 132, 692-731.

Gawronski, B., \& Cesario, J. (2013). Of mice and men: What animal research can tell us about context effects on automatic responses in humans. Personality and Social Psychology Review, 17, 187215.

Gawronski, B., \& De Houwer, J. (2014). Implicit measures in social and personality psychology. In $\mathrm{H}$. T. Reis, \& C. M. Judd (Eds.), Handbook of research methods in social and personality psychology (2nd edition, pp. 283-310). New York: Cambridge University Press.

Gawronski, B., Hu, X., Rydell, R. J., Vervliet, B., \& De Houwer, J. (2015). Generalization and contextualization in automatic evaluation revisited: A meta-analysis of successful and failed replications. Journal of Experimental Psychology: General, 144, e50-e64.

Gawronski, B., Rydell, R. J., Vervliet, B., \& De Houwer, J. (2010). Generalization versus contextualization in 
automatic evaluation. Journal of Experimental Psychology: General, 139, 683-701.

Gawronski, B., Ye, Y., Rydell, R. J., \& De Houwer, J. (2014). Formation, representation, and activation of contextualized attitudes. Journal of Experimental Social Psychology, 54, 188-203.

Gilbert, D. T., \& Malone, P. S. (1995). The correspondence bias. Psychological Bulletin, 117, 21-38.

Jenkins, L. J., Yang, Y.-J., Goh, J., Hong, Y.-Y., \& Park, D. C. (2010). Cultural differences in the lateral occipital complex while viewing incongruent scenes. Social Cognitive and Affective Neuroscience, 5, 236241.

Ji, L. J., Zhang, Z., \& Nisbett, R. E. (2004). Is it culture or is it language? Examination of language effects in cross-cultural research on categorization. Journal of Personality and Social Psychology, 87, 57-65.

Kashima, Y. (2015). Causal and meaning-based explanation. In B. Gawronski \& G. V. Bodenhausen (Eds.), Theory and explanation in social psychology (pp. 41- 62). New York: Guilford Press.

LeBel, E. P., \& Paunonen, S. V. (2011). Sexy but often unreliable: The impact of unreliability on the replicability of experimental findings with implicit measures. Personality and Social Psychology Bulletin, 37, 570-583.

Masuda, T., \& Nisbett, R. E. (2001). Attending holistically versus analytically: Comparing the context sensitivity of Japanese and Americans. Journal of Personality and Social Psychology, 81, 992-934.

Masuda, T., Russell, M. J., Chen, Y. Y., Hioki, K., \& Caplan, J. B. (2014). N400 incongruity effect in an episodic memory task reveals different strategies for handling irrelevant contextual information for Japanese than European Canadians. Cognitive Neuroscience, 5, 17-25.

Choi, I., \& Nisbett, R. E. (2000). Cultural psychology of surprise: Holistic theories and recognition of contradiction. Journal of Personality and Social Psychology, 79, 890-905.

Maxwell, S. E., Lau, M. Y., \& Howard, G. S. (2015). Is psychology suffering from a replication crisis? What does "failure to replicate" really mean? American Psychologist, 70, 487-498.

Open Science Collaboration. (2015). Estimating the reproducibility of psychological science. Science, 349(6251), aаc4716.

Oyserman, D., \& Lee, S. W. (2008). Does culture influence what and how we think? Effects of priming individualism and collectivism. Psychological Bulletin, 134, 311-342.
Payne, B. K., Cheng, S. M., Govorun, O., \& Stewart, B. D. (2005). An inkblot for attitudes: Affect misattribution as implicit measurement. Journal of Personality and Social Psychology, 89, 277-293.

Peng, K., \& Nisbett, R. E. (1999). Culture, dialectics, and reasoning about contradiction. American Psychologist, 54, 741-754.

Roese, N. J., \& Sherman, J. W. (2007). Expectancies. In E. T. Higgins \& A. W. Kruglanski (Eds.), Social psychology: Handbook of basic principles (2nd Ed., pp. 91-115). New York: Guilford Press.

Rydell, R. J., \& Gawronski, B. (2009). I like you, I like you not: Understanding the formation of contextdependent automatic attitudes. Cognition and Emotion, 23, 1118-1152.

Smith, E. R. (1996). What do connectionism and social psychology offer each other? Journal of Personality and Social Psychology, 70, 893-912.

Spencer-Rodgers, J., Williams, M. J., \& Peng, K. (2010). Cultural differences in expectations of change and tolerance for contradiction: A decade of empirical research. Personality and Social Psychology Review, 14, 296-312.

Stanley,D. J., \& Spence, J. R. (2014). Expectations for replications: Are yours realistic? Perspectives on Psychological Science, 9, 305-318.

Van Bavel, J. J., Mende-Siedlecki, P., Brady, W. J. \& Reinero, D. A. (2016). Contextual sensitivity in scientific reproducibility. Proceedings of the National Academy of Sciences, 113, 6454-6459.

Ye, Y., \& Gawronski. B. (in press). Contextualization of mental representations and evaluative responses: A theory-based analysis of cultural differences. In J. Spencer-Rodgers \& K. Peng (Eds.), The psychological and cultural foundations of East Asian cognition: Contradiction, change, and holism. New York: Oxford University Press.

Zhou, X., He, L., Yang, Q., Lao J., \& Baumeister, R. F. (2012). Control deprivation and styles of thinking. Journal of Personality and Social Psychology, 102, 460-478.

\section{Author Note}

The research reported in this article has been supported by grants from the Canada Research Chairs Program (215983) and the Social Sciences and Humanities Research Council of Canada (410-20110222) to the last author. We would like to thank Clara Chan, Joyce Chan, Jasmine Desjardins, Pearlene Ng, and Jolene Tan for their assistance in collecting the data.

Correspondence concerning this article should be sent to: Yang Ye, Department of Experimental-Clinical and Health Psychology, Ghent University, Henri Dunantlaan 2, B-9000 Gent, Belgium, Email: yang.ye@ugent.be 


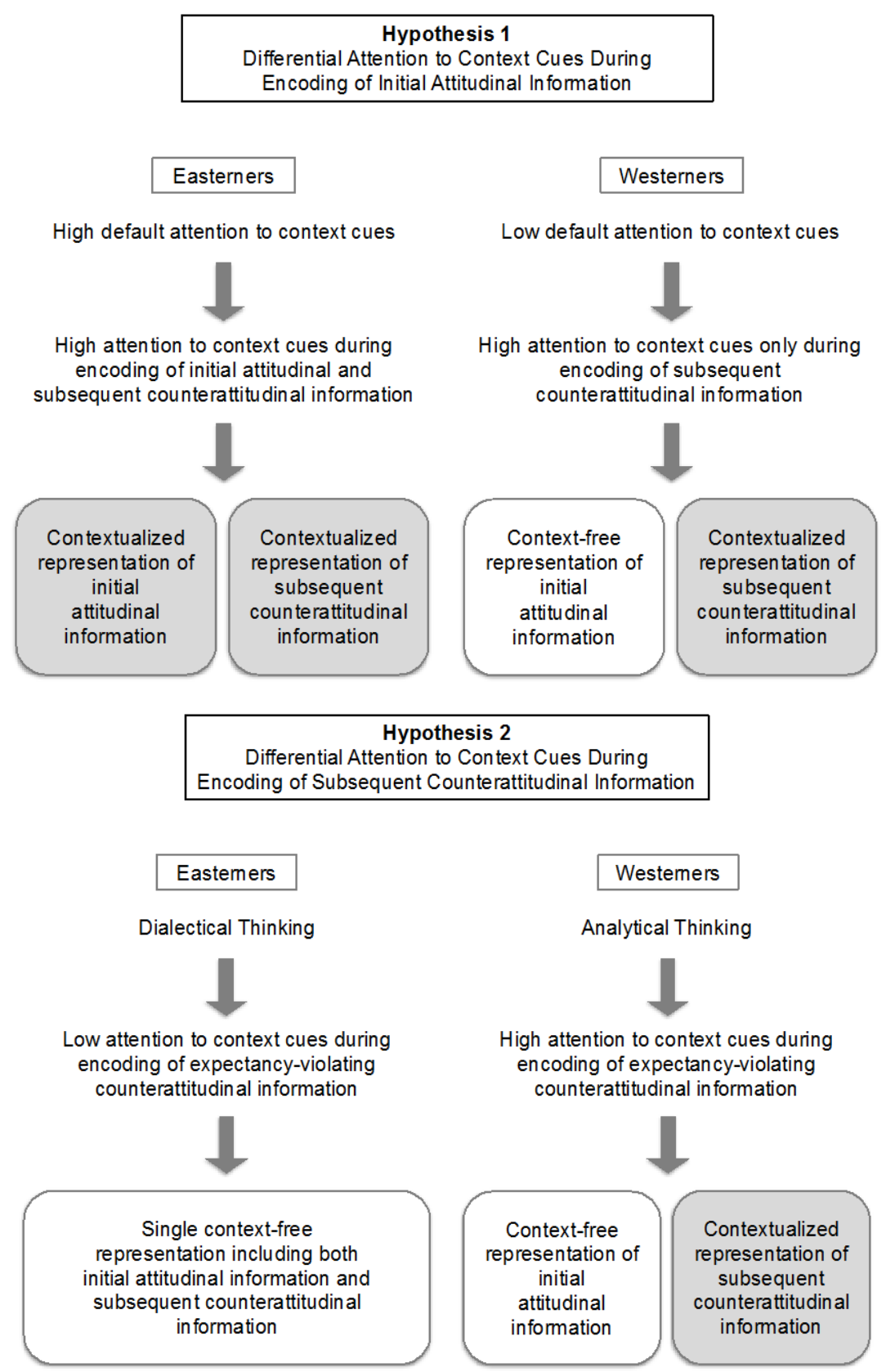

Figure 1. Hypotheses about East-West differences in attention to context during encoding of conflicting evaluative information and the formation of contextualized representations. 


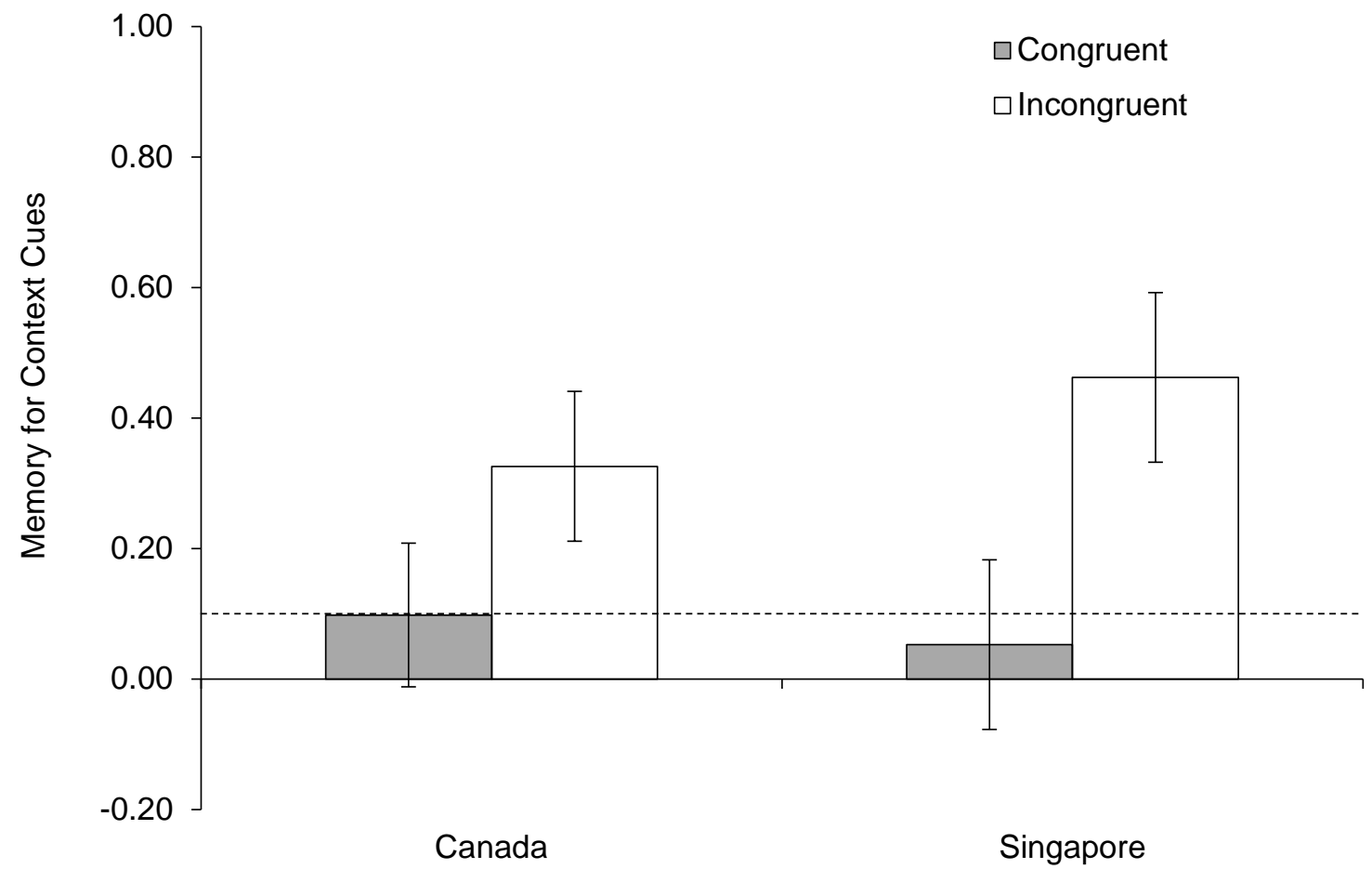

Figure 2. Proportion of correct memory for context cues as a function of target statement (congruent with initial information vs. incongruent with initial information) and country (Canada vs. Singapore), Study 1 . The dotted line depicts chance-level performance of 10\% correct memory judgments. Error bars depict $95 \%$ confidence intervals. 


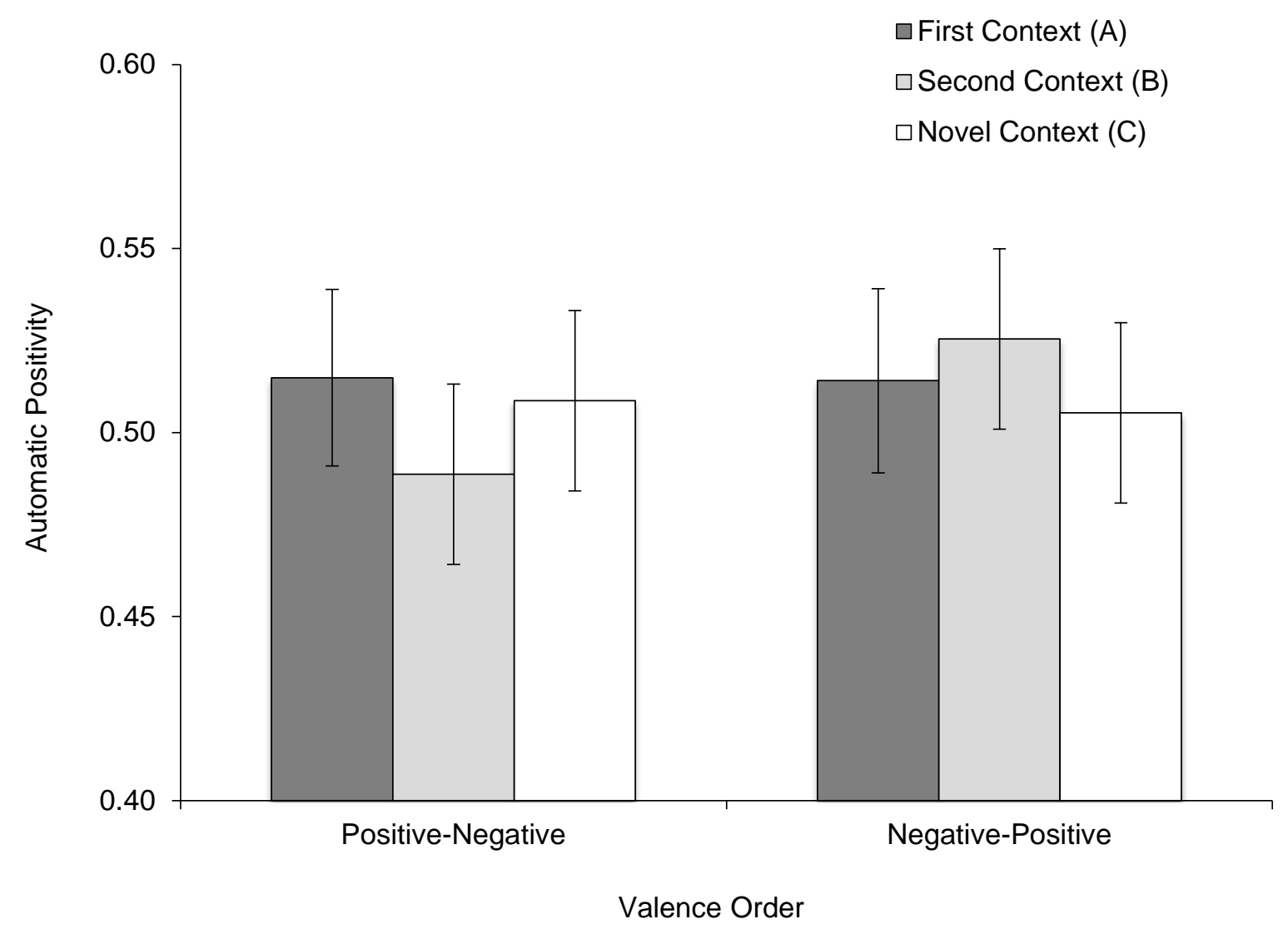

Figure 3. AMP scores of automatic evaluation as a function of Valence Order (positive-negative vs. negativepositive) and evaluation context (first context vs. second context vs. novel context), Study 2. Error bars depict 95\% confidence intervals. 